\title{
Apoptosis induction in human glioblastoma multiforme T98G cells upon temozolomide and quercetin treatment
}

\author{
Joanna Jakubowicz-Gil • Ewa Langner • Dorota Bądziul • \\ Iwona Wertel • Wojciech Rzeski
}

Received: 6 February 2013 / Accepted: 27 March 2013 /Published online: 12 April 2013

(C) The Author(s) 2013. This article is published with open access at Springerlink.com

\begin{abstract}
Glioblastoma multiforme is the most aggressive primary brain tumour. At the cellular and molecular levels, several mechanisms responsible for apoptosis or autophagy induction are blocked. Identification of molecular targets stimulating cells to initiate programmed cell death should be performed for therapeutic purposes. A promising solution is the combination of temozolomide and quercetin. The aim of our study was to evaluate the effect of both drugs, applied alone and in combinations, on apoptosis and autophagy induction in human glioblastoma multiforme T98G cells. Our results clearly indicate that quercetin and temozolomide induce apoptosis very significantly, having no effect on autophagy induction. At the molecular level, it was correlated with caspase 3 and 9 activation, cytochrome c release from the mitochondrium and a decrease in the mitochondrial membrane potential. Both drugs are also potent Hsp27 and Hsp72 inhibitors. This suggests that the apoptotic signal goes through an internal pathway. Increased expression of caspase 12 and the presence of several granules in the
\end{abstract}

J. Jakubowicz-Gil $(\bowtie) \cdot$ D. Bądziul

Department of Comparative Anatomy and Anthropology,

Maria Curie-Skłodowska University, Akademicka 19,

20-033 Lublin, Poland

e-mail: jjgil@poczta.umcs.lublin.pl

E. Langner $\cdot$ W. Rzeski

Department of Medical Biology, Institute of Agricultural

Medicine, Jaczewskiego 2, 20-950 Lublin, Poland

W. Rzeski

Department of Immunology and Virology,

Maria Curie-Skłodowska University, Akademicka 19,

20-033 Lublin, Poland

I. Wertel

1st Department of Gynecology, University School of Medicine,

Staszica 16, 20-081 Lublin, Poland cytoplasm after temozolomide treatment with or without quercetin preceding appearance of apoptosis may suggest that apoptosis is initiated by ER stress. Additionally, it was accompanied by changes in the nuclear morphology from circular to 'croissant like'.

Keywords Temozolomide · Quercetin · Apoptosis · Autophagy · ER stress · Glioblastoma multiforme

\section{Introduction}

Glioblastoma multiforme (grade IV) is the most common and the most aggressive primary brain tumour. It is ultimately recurrent. Despite the enormous progress in the development of surgical techniques and radio- and chemotherapy, treatment of malignant gliomas is extremely difficult and can extend patients' lives by only a few months. The average length of survival of a patient with this type of cancer has not improved significantly over the past few decades remaining at $12-15$ months. Only $3-5 \%$ of patients survive more than 3 years. The high invasiveness of glioma results from the capability of cancer cells of extensive proliferation and the 'diffuse infiltration' between normal brain cells, which makes them elusive targets for effective surgical management [1]. The problem of resistance of gliomas to treatment should also be analysed at the molecular level. In glioblastoma cells, several mechanisms responsible for programmed cell death induction like apoptosis or autophagy are blocked, while molecular chaperones promoting cell survival are overexpressed [2-4]. A better understanding of the complex biology of glioma cells is essential for identification of key molecular targets stimulating signalling pathways that lead to initiation of programmed cell death.

Temozolomide is a new drug in glioma therapy, which alone or in combination with radiotherapy prolongs the 
median survival time. The cytotoxicity of temozolomide is due to formation of $\mathrm{O}^{6}$-methylguanine in DNA which mispairs with thymine during the next cell cycle of DNA replication. In consequence, glioma cells respond by undergoing $\mathrm{G}_{2} / \mathrm{M}$ arrest and ultimately die by autophagy or apoptosis [5-9]. Our earlier experiments conducted on the anaplastic astrocytoma cell line MOGGCCM revealed that the anticancer properties of temozolomide are potentiated by quercetin (3, 3', 4',5,7-pentahydroxyflavone), a natural flavonoid found in a broad range of fruits and vegetables, e.g. apples, grapes, lemons, onion, kale or tomatoes, with the daily intake of about $30 \mathrm{mg}$. It has multiple biological, pharmacological and medical applications. It is one of the most potent antioxidants. Quercetin also facilitates apoptosis of tumour cells by activation of caspase 3 and caspase 9 and cytochrome c release. It is also a well-known inhibitor of heat shock protein expression [10-16].

Therefore, the aim of our study was to investigate the effect of temozolomide and quercetin applied alone or in combination on apoptosis and autophagy induction in the human glioblastoma multiforme T98G cell line. We analysed the morphology typical of programmed cell death as well as the molecular mechanism underlying this process based on Hsp27, Hsp72, cytochrome c, beclin 1, caspase 12 protein expression and caspase 3, caspase 8 and caspase 9 activity. Analyses of the shape of the cell nuclei as a hallmark of glioblastoma were also performed.

\section{Materials and methods}

\section{Cells and culture conditions}

The human glioblastoma multiforme cells (T98G, European Collection of Cell Cultures) were grown in a 3:1 mixture of DMEM and nutrient mixture F-12 Ham (Ham's F-12) (Sigma) supplemented with $10 \%$ FBS (Sigma), penicillin (100 U/ml) (Sigma) and streptomycin $(100 \mu \mathrm{g} / \mathrm{ml})$ (Sigma). The cultures were kept at $37^{\circ} \mathrm{C}$ in humidified atmosphere of $95 \%$ air and $5 \% \mathrm{CO}_{2}$.

Drug treatment

Temozolomide (TMZ, Schering-Plough) at the final concentrations of $1,5,10,25,50,100,150$ and $200 \mu \mathrm{M}$ and quercetin $\left(3,3^{\prime}, 4^{\prime}, 5,7\right.$-pentahydroksyflavone) (Sigma) at the final concentrations of $1,5,10,25,50$ and $100 \mu \mathrm{M}$ were used in the experiments. The drugs were dissolved in dimethyl sulfoxide (DMSO, Sigma). The final concentration of DMSO in the culture medium did not exceed $0.01 \%$, which as indicated in preliminary experiments did not influence cell viability and the expression of the proteins studied. Three variants of drug treatment were performed. In the first one, the cells were incubated only with quercetin or temozolomide for 24,48 or $72 \mathrm{~h}$. In the second variant, quercetin and temozolomide were added to the culture medium simultaneously and incubated for 24 or $48 \mathrm{~h}$. In the third one, the cells were pre-incubated with one of the two drugs for 6 or $24 \mathrm{~h}$, followed by treatment with the other drug for the next 18 or $24 \mathrm{~h}$. As a control, the cells were incubated with $0.01 \%$ of DMSO.

Detection of apoptosis and necrosis with fluorochromes

For identification of apoptosis and necrosis, the cells were stained with a mixture of fluorescent dyes Hoechst 33342 (Sigma) and propidium iodide (Sigma), respectively [17]. The morphological analysis was performed under a fluorescence microscope (Nikon E-800). Cells exhibiting blue fluorescent nuclei (fragmented and/or with condensed chromatin) were interpreted as apoptotic. Cells exhibiting pink fluorescent nuclei were interpreted as necrotic. At least 1,000 cells in randomly selected microscopic fields were counted under a microscope. Each experiment was performed in triplicate.

Detection of acidic vesicular organelles with acridine orange

Autophagy is a process of sequestrating cytoplasmic proteins into the lytic compartment and is characterised by formation and promotion of acidic vesicular organelles (AVOs). Vital staining with $1 \mu \mathrm{g} / \mathrm{ml}$ of acridine orange (AO) for $15 \mathrm{~min}$ was performed for detection of AVOs in the cells treated with quercetin and/or temozolomide [18]. Typical AO-positive cells exhibiting granular discretion of AVOs in the cytoplasm were interpreted as autophagic. A morphological analysis was performed under a fluorescence microscope (Nikon E-800). At least 1,000 cells in randomly selected microscopic fields were counted. Each experiment was performed in triplicate. The percentage of autophagic cells was calculated as the number of cells with AVOs versus the total number of stained cells.

Detection of apoptosis and necrosis by flow cytometry

The Annexin V-FITC apoptosis detection kit (Sigma) was used for detection of apoptosis and necrosis by flow cytometry. The samples were prepared according to the manufacturer's protocol. Briefly, the control and drug-treated cells were incubated with $5 \mu \mathrm{l}$ of Annexin V-FITC and $10 \mu \mathrm{l}$ of propidium iodide for $10 \mathrm{~min}$. Immediately after staining, the cells were analysed on the FacsCanto instrument (Becton Dickinson, San Jose, CA, USA). A total of 30,000 events were acquired and analysed using FacsDiva software. Cells which were in the early stage of the apoptotic process were stained only with Annexin V-FITC, whereas late apoptotic 
cells were stained by both fluorochromes. Cells stained only with propidium iodide were interpreted as necrotic. Live cells showed no staining by either propidium iodide or Annexin V-FITC. Each experiment was performed in triplicate.

\section{Mitochondrial membrane potential}

Fluorochrome 3,3'-dihexyloxacarbocyanine iodide $\mathrm{DiOC}_{6}(3)$ was used for studying the changes in the mitochondrial membrane potential $\left(\Delta \psi_{\mathrm{m}}\right)$ in cells incubated with quercetin and temozolomide. At low concentrations, it accumulates in the mitochondria. Loss in $\mathrm{DiOC}_{6}(3)$ staining indicates disruption of the mitochondrial inner transmembrane potential [19]. The control and drug-treated cells were incubated with the fluorochrome at the final concentration of $50 \mathrm{nM}$ for $20 \mathrm{~min}$ at $37^{\circ} \mathrm{C}$ in the dark, washed three times with PBS and subjected to the FacsCanto instrument (Becton Dickinson). A total of 30,000 events were acquired and analysed using FacsDiva software. Each experiment was performed in triplicate.

\section{Isolation of the mitochondrial fraction}

A commercial isolation kit was used (Sigma) for the isolation of the mitochondria. The samples were prepared according to the manufacturer's protocol. Briefly, after quercetin or/and temozolomide treatment, the cells at the density about $2 \times 10^{7}$ were trypsinised, centrifuged for $5 \min (600 \times \mathrm{g})$ and washed twice with PBS. The cell pellet was resuspended in lysis buffer (Sigma), incubated for $5 \mathrm{~min}$ on ice and centrifuged for $10 \mathrm{~min}(600 \times g)$. The supernatant was transferred onto a fresh tube and centrifuged at $11,000 \times g$ for $10 \mathrm{~min}$. The pellet was resuspended in cell lysis buffer and used for electrophoresis.

\section{Isolation of the cytosolic fraction}

After quercetin and/or temozolomide treatment, the cells were lysed in hot SDS-loading buffer $(125 \mathrm{mM}$ Tris- $\mathrm{HCl}$ pH 6.8; $4 \%$ SDS; $10 \%$ glycerol; 100 mM DTT), boiled in a water bath for $10 \mathrm{~min}$ and centrifuged at $10,000 \times \mathrm{g}$ for $10 \mathrm{~min}$, and the supernatants were collected. The protein concentration was determined by the Bradford method [20] and samples of the supernatants containing $80 \mu \mathrm{g}$ of proteins were used for electrophoresis.

\section{Immunoblotting}

Cytoplasmic and mitochondrial samples were separated by $10 \%$ SDS-polyacrylamide gel electrophoresis [21] and subsequently transferred onto Immmobilon $\mathrm{P}$ membrane (Sigma). Following the transfer, the membrane was blocked with $3 \%$ low fat milk in PBS for $1 \mathrm{~h}$ and incubated overnight with mouse anti-Hsp72 monoclonal antibody (SPA 810, StressGen) diluted 1:1,000, anti-Hsp27 (SPA 800, StressGen) diluted 1:1,000, rabbit beclin 1 antibody (Sigma) diluted 1:500, anti-caspase 12 (Cell Signaling) diluted 1:1,000 and sheep anti-cytochrome $\mathrm{c}$ antibody (Sigma) diluted 1:1,000. The membranes were washed three times for 10 min with PBS containing $0.05 \%$ Triton X-100 (Sigma) and incubated for $2 \mathrm{~h}$ with a 1:30,000 dilution of alkaline phosphatase-conjugated goat anti-mouse IgG, antisheep IgG or anti-rabbit IgG (Sigma). The membranes were visualised with alkaline phosphatase substrate (5-bromo-4chloro-3-indolylphosphate and nitro-blue tetrazolium, Sigma) in colour development buffer (DMF, Sigma). The data were normalised relative to $\beta$-actin (Sigma, working dilution 1:2,000, data not shown).

The levels of protein expression were determined using the Bio-Profil Bio-1D Windows Application V.99.03 programme. Three independent experiments were performed.

\section{Caspase activity assay}

Caspases are cysteine proteases, which in normal conditions exist as inactive pro-forms or zymogens. They are cleaved to the active form following induction of apoptosis. The activity of caspases 3, 8 and 9 was estimated using the SensoLyte ${ }^{\circledR}$ AMC Caspase Substrate Sampler Kit (AnaSpec) in the control and drug-treated cells. Sample preparation and enzymatic reaction were performed according to the manufacturer's protocol. The fluorescence of AMC was monitored at $\mathrm{Ex} / \mathrm{Em}=354 \mathrm{~nm} / 442 \mathrm{~nm}$ in 96 -well black microplates using 2030 Multilabel Reader Victor ${ }^{\mathrm{TM}} \mathrm{x} 4$ (Perkin Elmer).

\section{Nuclear morphology}

For the analysis of the nuclear shape in the control and drugtreated cells, the roundness factor was used [22, 23] with value 1 for a circle and smaller values for increasing irregularities of nuclear shape. For visualisation of the nucleus, the cells were stained with Hoechst 33342 at a final concentration of $80 \mu \mathrm{g} / \mathrm{ml}$ for $5 \mathrm{~min}$. The measurement of the shape of the nuclei was performed with the Image J programme on digital microscopic images, projected on the computer screen. The tumour cell nuclei were manually traced. A minimum of 300 tumour nuclei per experimental variant were analysed. Three experimental variants were performed.

\section{ER staining}

For identification of ER, a staining method with fluorochrome 3,3'-dihexyloxacarbocyanine iodide was used [24]. The cells were incubated with $10 \mu \mathrm{M}$ of $\operatorname{DiOC}_{6}(3)$ for $10 \mathrm{~min}$ in the dark at $37{ }^{\circ} \mathrm{C}$. Morphological analysis was performed under a fluorescent microscope Nikon E-800. 
Statistical analysis

The data are presented as mean \pm standard deviation (SD). The statistical evaluation was performed with a one-way ANOVA test followed by Dunnett's multiple comparison test. $P<0.05$ was taken as the criterion of significance.

\section{Results}

Sensitivity of T98G cells to temozolomide or quercetin treatment

To estimate the sensitivity of T98 $\mathrm{G}$ cells to temozolomide or quercetin treatment, staining was performed with dyes specific for apoptosis, necrosis and autophagy: Hoechst 33342, propidium iodide and acridine orange, respectively (Figs. 1 and 2). Microscopic observations of stained cells revealed that temozolomide was very effective in apoptosis induction (Fig. 1a-c). A significant increase in the number of programmed dead cells was observed after $24 \mathrm{~h}$ of incubation, reaching a maximum (12\%) at the concentration of 100 and $150 \mu \mathrm{M}$ (Fig. 1a). The longer incubation time was even more effective, and signs of apoptotic cell death were noticed in about $22 \%$ of cells after $48 \mathrm{~h}$ of incubation with temozolomide $150 \mu \mathrm{M}$ (Fig. 1b) and $35 \%$ after $72 \mathrm{~h}$ of treatment with the drug concentration of $100 \mu \mathrm{M}$ (Fig. 1c). Besides apoptosis, temozolomide applied to the culture medium for 48 and $72 \mathrm{~h}$ at concentrations higher than $100 \mu \mathrm{M}$ induced necrosis in T98G cells. What was also interesting, the increased number of necrotic cells was accompanied by a decreased number of apoptotic ones, when the cells were incubated with temozolomide starting with $200 \mu \mathrm{M}$ applied for $48 \mathrm{~h}$ and $150 \mu \mathrm{M}$ after $72 \mathrm{~h}$ of treatment. Temozolomide had no effect on autophagy induction.
Similar to temozolomide, quercetin was also effective in apoptosis induction, but in comparison to the alkylating drug, its pro-apoptotic properties were weaker (Fig. 2a-c). Quercetin induced apoptosis very effectively after the 48-hlong treatment with the concentrations of $25(14 \%)$ and $50 \mu \mathrm{M}(22 \%)$ (Fig. 2b, c). The longer period of incubation with the higher flavonoid concentration induced mainly necrosis (data not shown). The necrotic type of cell death also dominated after the 24-h-long incubation with the concentration above $100 \mu \mathrm{M}$ (Fig. 2a). Similar to TMZ, quercetin had no significant effect on autophagy induction.

Sensitivity of T98G cells to combined temozolomide and quercetin treatment

To estimate the combined effect of quercetin and temozolomide on cell death, two possible variants of the drug treatment were performed. In the first variant, the cells were incubated simultaneously with both drugs, and in the second one, the cells were pre-incubated with one of the drugs followed by incubation with the other one. The type of death was estimated through observation of cells after staining with Hoechst 33342, acridine orange and propidium iodide (Fig. 3). Incubation of T98G cells with temozolomide at the concentration of $50 \mu \mathrm{M}$ with increasing quercetin concentrations $(0-50 \mu \mathrm{M})$ for 24 (Fig. 3a-c) and $48 \mathrm{~h}$ (Fig. 3d-f) revealed that such a combination induced mainly apoptosis and the effectiveness of such a treatment was time and concentration dependent. The number of apoptotic cells rose gradually with the increased concentration of quercetin, being the most effective in a combination containing $50 \mu \mathrm{M}$ of the flavonoid. Increased effectiveness was observed after the longer incubation time. When T98G cells were treated simultaneously with the combination of $50 \mu \mathrm{M}$ of quercetin and $50 \mu \mathrm{M}$ of temozolomide for $24 \mathrm{~h}$, apoptosis was observed in $6 \%$ of the cells, while after the 48 -h-long incubation,
Fig. 1 The level of apoptosis, necrosis and autophagy induction in the glioblastoma multiforme T98G cell line treated with temozolomide $(0-200 \mu \mathrm{M})$ for 24 (a), 48 (b) and $72 \mathrm{~h}(\mathbf{c})$, stained with Hoechst 33342, propidium iodide and acridine orange. $* P<0.05$ a

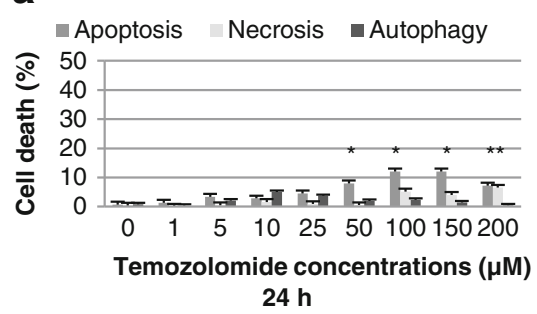

C

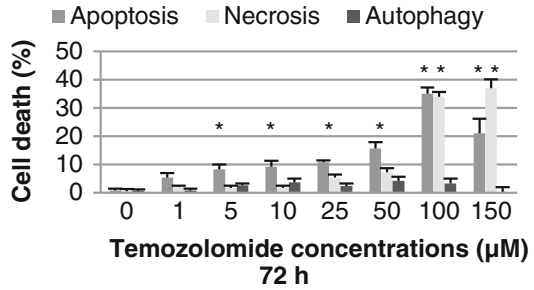

b

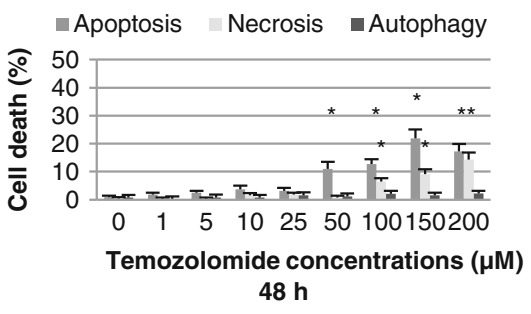


Fig. 2 The time-dependent effect of quercetin $(0-100 \mu \mathrm{M})$ on apoptosis, necrosis and autophagy induction in the T98G cell line incubated with the drug for 24 (a) and $48 \mathrm{~h}$ (b). c A picture of $\mathrm{T} 98 \mathrm{G}$ cell stained with Hoechst 33342 and propidium iodide after $48 \mathrm{~h}$ of treatment with $50 \mu \mathrm{M}$ of quercetin. Green arrow indicates necrotic cell, while red arrow points apoptotic cell. ${ }^{*} P<0.05$ a

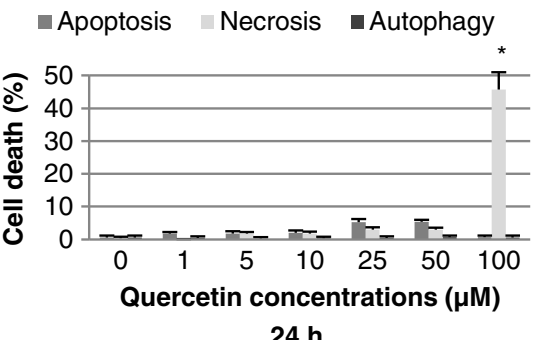

b
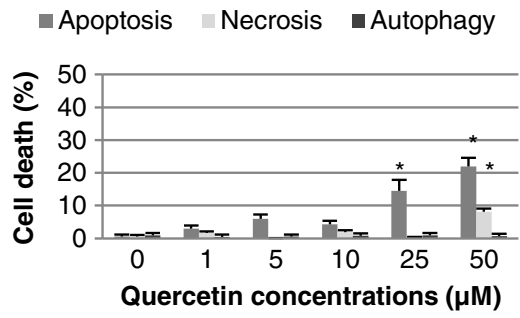

$48 \mathrm{~h}$

C

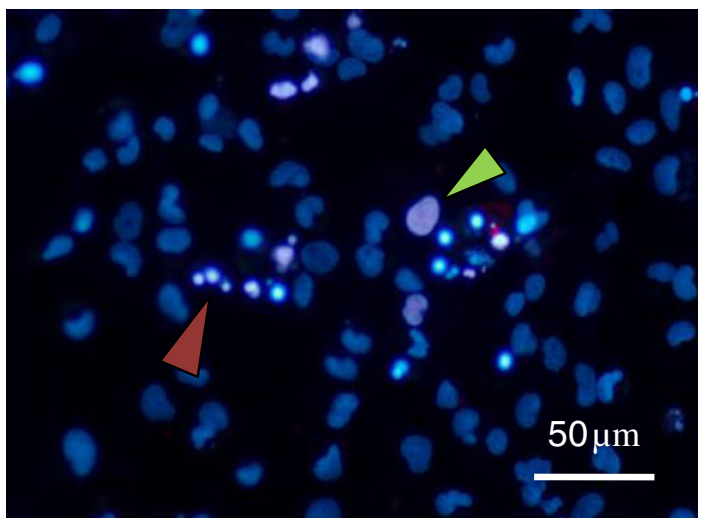

apoptosis was observed in $18 \%$ of the cells. Although the percentage of apoptotic cells after simultaneous drug application was similar to the level observed after separate quercetin treatment, the number of necrotic cells was significantly lower

Fig. 3 Estimation of the level of apoptosis, necrosis and autophagy in T98G cells incubated with $50(\mathbf{a}-\mathbf{f})$ or $100 \mu \mathrm{M}(\mathbf{g}-\mathbf{i})$ of temozolomide and an increasing quercetin concentration $(0-50 \mu \mathrm{M})$ for $24 \mathrm{~h}(\mathbf{a}-\mathbf{c}, \mathbf{g}-\mathbf{i})$ and $48 \mathrm{~h}(\mathbf{d}-\mathbf{f})$, stained with Hoechst 33342 and propidium iodide; $\mathbf{a}, \mathbf{d}, \mathbf{g}$ cells pre-incubated with quercetin followed by temozolomide administration; $\mathbf{b}, \mathbf{e}, \mathbf{h}$ cells treated with both drugs administered at the same time; c, $\mathbf{f}, \mathbf{i}$ cells pre-incubated with temozolomide followed by quercetin administration a

apoptosis $\backsim$ Necrosis $\backsim$ Autophagy

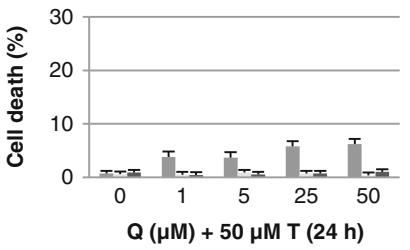

d

$₫$ Apoptosis Necrosis $\backsim$ Autophagy

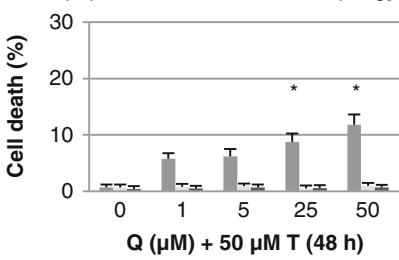

g

Apoptosis Necrosis $\approx$ Autophagy

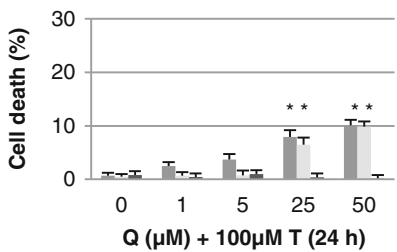

when flavonoid was accompanied by temozolomide. In the case of separate quercetin treatment, the level of necrosis was high and reached $10 \%$. When flavonoid was combined with temozolomide, the percentage of necrotic cells was about 2.

b

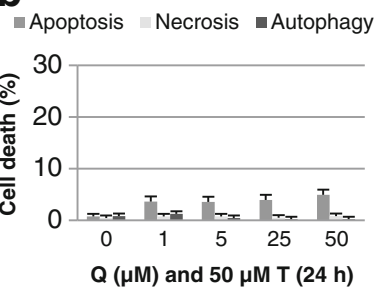

e

Apoptosis Necrosis -Autophagy

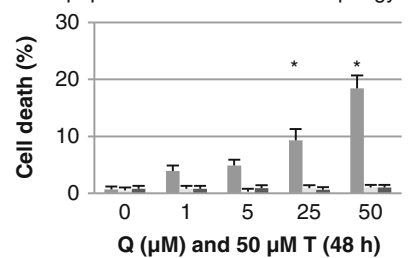

h

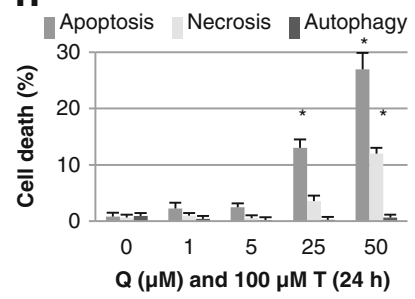

C

Apoptosis Necrosis $\square$ Autophagy

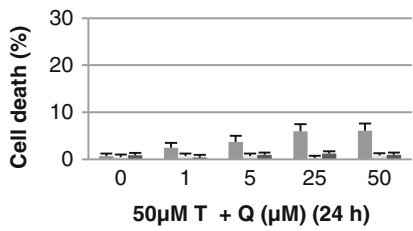

f

Apoptosis Necrosis $\backsim$ Autophagy
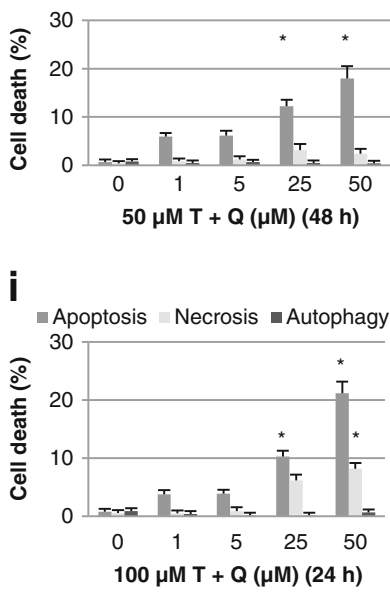
When the T98G cells were treated with $100 \mu \mathrm{M}$ of TMZ combined with different quercetin concentrations for $24 \mathrm{~h}$, the number of apoptotic cells was higher than that observed after incubation with $50 \mu \mathrm{M}$ of the alkylating drug (Fig. 3g-i). Application of $100 \mu \mathrm{M} \mathrm{TMZ}$ and $50 \mu \mathrm{M}$ of quercetin induced apoptosis in $27 \%$ of cells; unfortunately, it was accompanied by a significant necrosis, and after $48 \mathrm{~h}$ of incubation, necrosis was dominant (data not shown). Therefore, temozolomide and quercetin at the concentration of $50 \mu \mathrm{M}$ applied to the culture medium for $48 \mathrm{~h}$ were chosen for further experiments. Considering the precedence of drug addition, the simultaneous treatment and pre-incubation variant with temozolomide were the most effective. Pre-incubation of cells with quercetin followed by the TMZ treatment was less effective. Incubation with both drugs had no effect on autophagy induction.

Evaluation of cell death and the mitochondrial transmembrane potential by flow cytometry

To confirm the reliability of the results obtained from the microscopic observations, the T98G cells treated with quercetin $(50 \mu \mathrm{M})$ and temozolomide $(50 \mu \mathrm{M})$ separately as well as in combination of both drugs for $48 \mathrm{~h}$ were analysed by flow cytometry according to the type of cell death (apoptosis or necrosis) and mitochondrial membrane potential.

Incubation of T98G cells with temozolomide resulted in significant induction of apoptosis (Table 1). Early apoptosis was observed in $3.82 \%$ of the cells, while late apoptosis was observed in $9.16 \%$. Quercetin induced programmed cell death in $16.74 \%$ of the cells, where $8.18 \%$ were at the early stage of the apoptotic process and $8.56 \%$ at the late one. The combination of both drugs was more effective and the percentage of apoptotic cells reached $22.75(11.75 \%$ for early apoptosis and $11 \%$ for late apoptosis) in the case of

Table 1 The effect of 48-h-long incubation of T98G cells with quercetin $(\mathrm{Q}, 50 \mu \mathrm{M})$ and/or temozolomide $(\mathrm{T}, 50 \mu \mathrm{M})$ on apoptosis and necrosis induction

\begin{tabular}{llll}
\hline & Necrosis (\%) & Early apoptosis (\%) & Late apoptosis (\%) \\
\hline Control & $0.25 \pm 0.07$ & $0.75 \pm 0.2$ & $0.14 \pm 0.1$ \\
Q & $2.7 \pm 0.4$ & $8.18 \pm 1.2^{*}$ & $8.56 \pm 0.07^{*}$ \\
T & $0.5 \pm 0.14$ & $3.82 \pm 0.14$ & $9.15 \pm 0.2^{*}$ \\
QT & $0.8 \pm 0.07$ & $9.95 \pm 0.49^{*}$ & $8.2 \pm 0.42^{*}$ \\
Q+T & $0.8 \pm 0.0$ & $11.75 \pm 0.07^{*}$ & $11 \pm 1^{*}$ \\
TQ & $0.8 \pm 0.14$ & $11.26 \pm 0.77^{*}$ & $10.3 \pm 0.6^{*}$ \\
\hline
\end{tabular}

Samples were analysed by flow cytometry with Annexin V-FITC detection kit. Each experiment was performed in triplicate

$Q T$ cells pre-incubated with quercetin, $Q+T$ simultaneous drug treatment, $T Q$ pre-incubation with temozolomide

$* P<0.05$ simultaneous application. A similar effect was observed when the T98G cells were pre-incubated with temozolomide followed by quercetin treatment $(21.8 \%)$. In the case of quercetin pre-incubation, the number of dead cells was slightly lower but still reached $17.8 \%$.

It is known that a decreased mitochondrial membrane potential is a good indicator of apoptotic cell death [4]. In our experiments, both quercetin and temozolomide applied alone or in combination decreased this potential (Fig. 4). The lowest value was observed after treatment with the simultaneous application of quercetin and temozolomide.

The effect of temozolomide and quercetin on the expression of marker proteins

Apoptosis and autophagy at the molecular level are characterised by changes in the expression of typical marker proteins. Using the immunoblot technique, we studied the effect of temozolomide and quercetin applied separately or in combinations (Fig. 5) on the level of expression of proapoptotic cytochrome c [in the cytoplasmic (Fig. 5c] and mitochondrial (Fig. 5d) fractions], anti-apoptotic molecular chaperones Hsp27 (Fig. 5b) and Hsp72 (Fig. 5a) and proautophagic beclin 1 (Fig. 5g). Additionally, we analysed fluorimetrically the activity of caspases 3,8 and 9, i.e. aspartate-specific cysteine proteases being part of the caspase cascade system, which plays a vital role in apoptosis (Fig. 5f). Using the immunoblot technique, we also studied the level of expression of caspase 12, a well-known enzyme mediating apoptosis under ER stress (Fig. 5e).

The quantitative and qualitative analyses of the immunoblots revealed that quercetin and temozolomide inhibited Hsp72 and Hsp27 expression in all the experimental variants. The studied drugs applied alone or in combinations decreased the level of cytochrome $\mathrm{c}$ in the mitochondrial fraction, which was accompanied by increased accumulation of the protein in the cytoplasm. Quercetin and temozolomide had no significant effect on the expression of the autophagy marker beclin 1 as its level was similar to

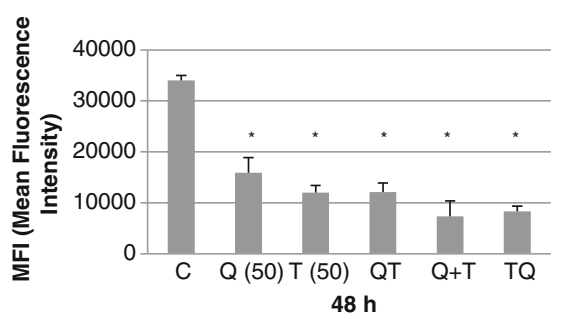

Fig. 4 The mitochondrial membrane potential in T98G cells stained with $\mathrm{DiOC}_{6}(3)$, incubated separately with $50 \mu \mathrm{M}$ of quercetin (Q) and $50 \mu \mathrm{M}$ of temozolomide $(\mathrm{T})$ or in combination of both drugs for $48 \mathrm{~h}$ and analysed by flow cytometry. $C$ control cells, $Q T$ cells pre-incubated with quercetin, $Q+T$ simultaneous drug treatment, $T Q$ pre-incubation with temozolomide. $* P<0.05$ 
a
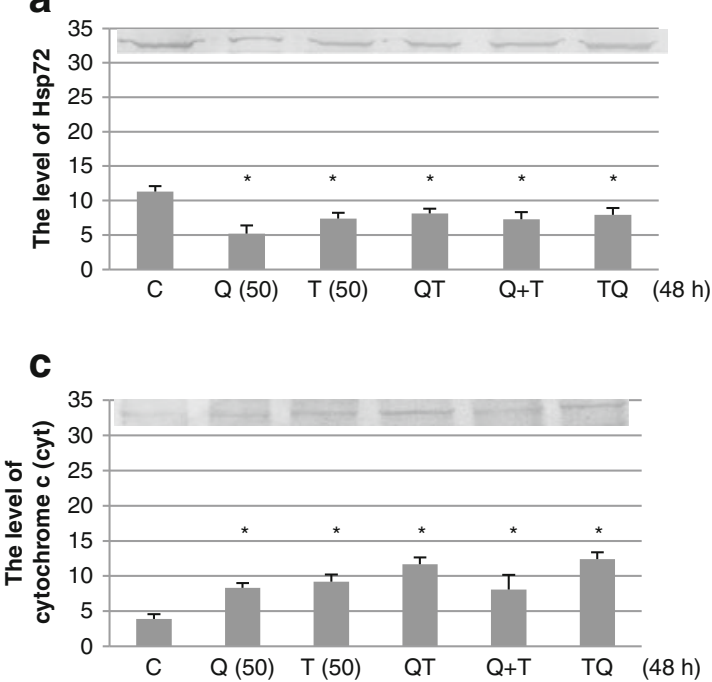

e
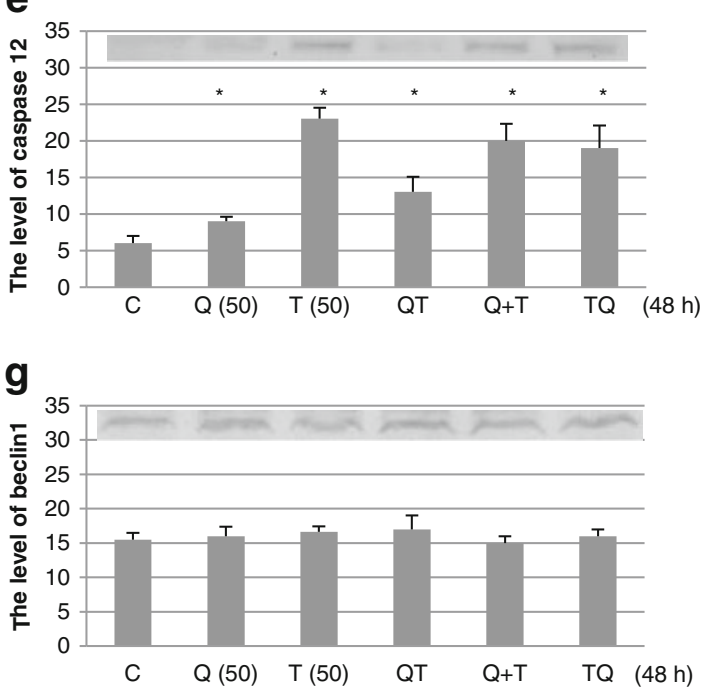

Fig. 5 Level of Hsp72 (a), Hsp27 (b), cytochrome c (c cytoplasmic, d mitochondrial fraction), caspase 12 (e) and beclin 1 (g) expression with representative blots and the activity of caspases 3,8 and 9 (f) after

the control one in all the experimental variants. In the case of caspases, increased expression of caspase 12 was observed after treatment with temozolomide separately as well as in combination with quercetin. Both drugs applied alone or in combination significantly increased the activity of caspase 3 and caspase 9 . The simultaneous drug treatment was the most potent. TMZ applied separately as well as in combination with quercetin did not change the caspase 8 activity. Its inhibition was observed after the quercetin treatment.

The effect of temozolomide and quercetin on the shape of nuclei and cytoplasm organisation

Staining of the cells with Hoechst 33342 revealed that temozolomide alone and together with quercetin changed
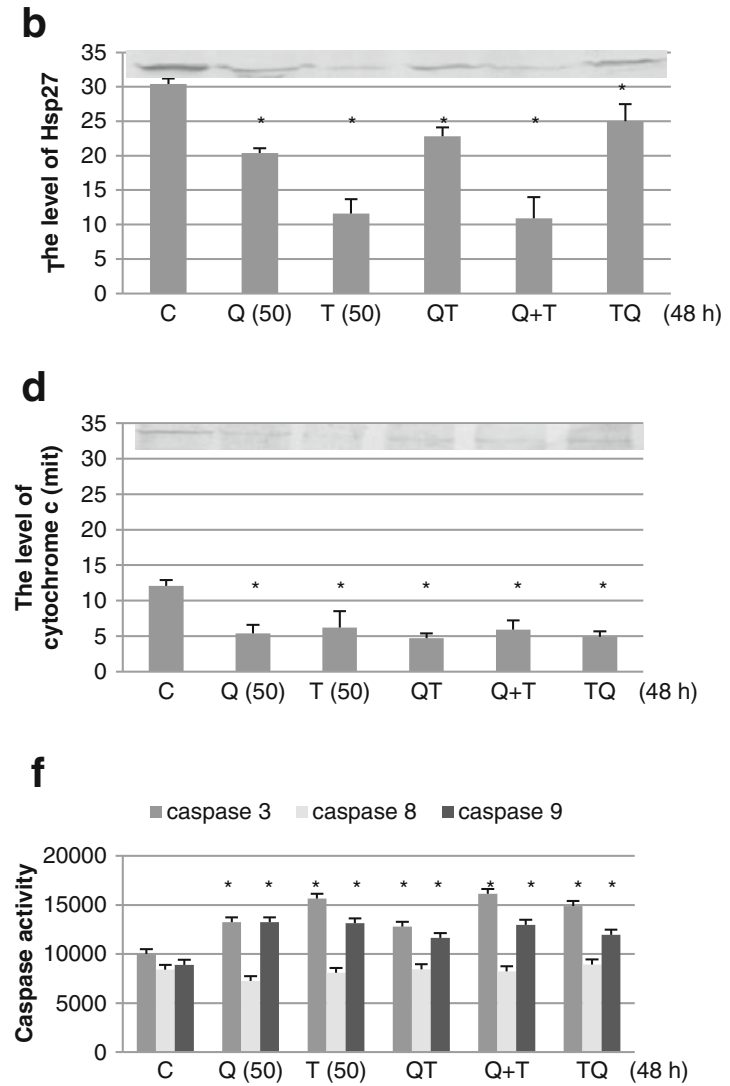

temozolomide (T) and quercetin (Q) treatment for $48 \mathrm{~h}$. $C$ control cells, $Q T$ cells pre-incubated with quercetin, $Q+T$ simultaneous drug treatment, $T Q$ pre-incubation with temozolomide. ${ }^{*} P<0.05$

the morphology of glioblastoma nuclei from circular, as observed in the control (Fig. 6a), to a more curved 'croissant-like' shape (Fig. 6b). The mean roundness factor used for the histomorphometrical analysis of the shape of the nuclei after TMZ with or without the flavonoid decreased from 0.86 (control) to about 0.6 (Fig. 6c). Quercetin did not change the shape of the nuclei and the mean roundness factor was similar to that in the control (0.85) (Fig. 6c). Staining of the T98G cells treated with temozolomide with orange acridine facilitated distinguishing round-shaped granules, which were not stained with the fluorochrome used (Fig. 7). The percentage of cells where such structures were noticed was the highest after $24 \mathrm{~h}$ of incubation (11\%) and decreased gradually with a longer incubation time. Granules were observed in about $9 \%$ of cells after a 48 -h- 
Fig. 6 The effect of temozolomide (T) and quercetin (Q) on the shape of the nuclei expressed by the mean roundness factor (MRF) after staining with Hoechst 33342. a Control cells, b nuclei after treatment with quercetin and temozolomide, c quantitative analysis. QT cells pre-incubated with quercetin, $Q+T$

simultaneous drug treatment, $T Q$ pre-incubation with temozolomide. ${ }^{*} P<0.05$ a

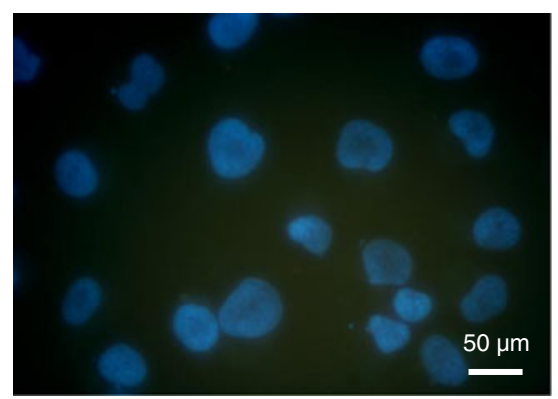

b

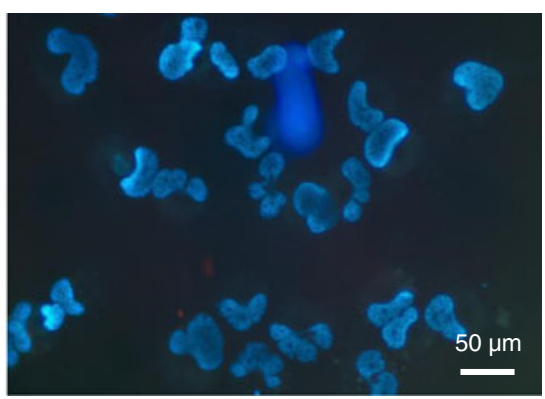

C

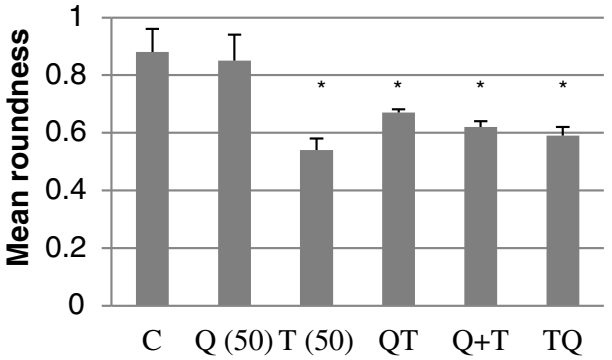

long incubation and $6 \%$ after $72 \mathrm{~h}$ after incubation with only TMZ and simultaneously with quercetin (Fig. 7a, b, d). No granules in the cytoplasm were noticed in the control nontreated cells and cells incubated with quercetin (data not shown). Additional staining of the T98G cells treated with the alkylating drug with or without flavonoid with $\mathrm{DiOC}_{6}(3)$, a fluorochrome that binds to ER, revealed the presence of round bulky structures within the reticulum that were similar in shape and size to the granules observed in the cytoplasm (Fig. 7d).
Localisation of Hsp27 and Hsp72 in T98G cells

The indirect immunofluorescence technique used to examine the localisation of Hsp27 (Fig. 8b, d) and Hsp72 (Fig. 8a, c) revealed cytoplasmic distribution of both proteins in the untreated cells as well as after temozolomide and quercetin application. On the basis of the colour and light intensity of the fluorochrome conjugated with the secondary antibody, it was noticed that the localisation of both proteins in the cytoplasm was different. The proteins in the control
Fig. 7 The effect of

temozolomide $(\mathrm{T})$ and quercetin (Q) on the cytoplasmic granule formation (white arrows) in the T98G cells. a Quantitative analysis, $\mathbf{b}$ cells stained with OA after temozolomide treatment, $\mathbf{c}$ ER structure in control cells stained with $\mathrm{DiOC}_{6}(3), \mathbf{d}$ ER structure in cells treated with temozolomide and stained with $\mathrm{DiOC}_{6}(3) . Q T$ cells pre-incubated with quercetin, $Q+T$ simultaneous drug treatment, $T+Q$ preincubation with temozolomide. ${ }^{*} P<0.05$ a

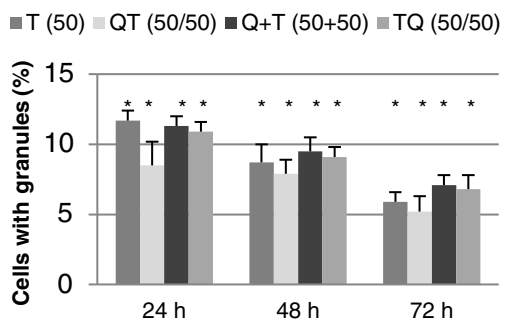

C

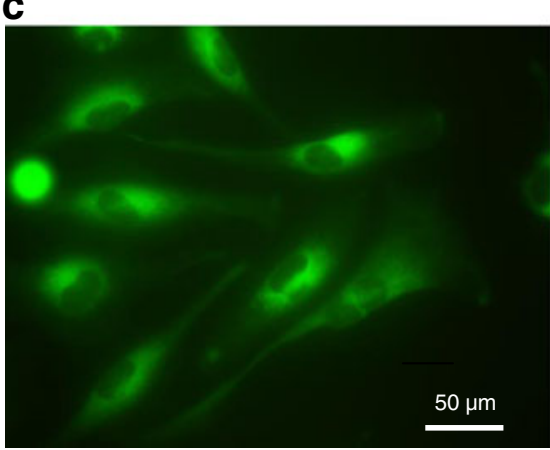

b

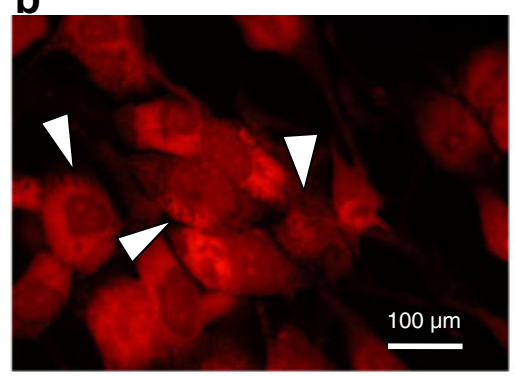

d

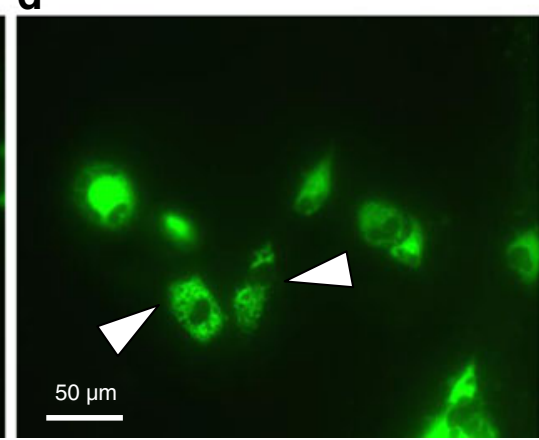


Fig. 8 The effect of temozolomide and quercetin on Hsp72 (a, c) and Hsp27 (b, d) in untreated cells $(\mathbf{a}, \mathbf{b})$ or treated with both drugs $(\mathbf{c}, \mathbf{d})$ for $48 \mathrm{~h}$ a

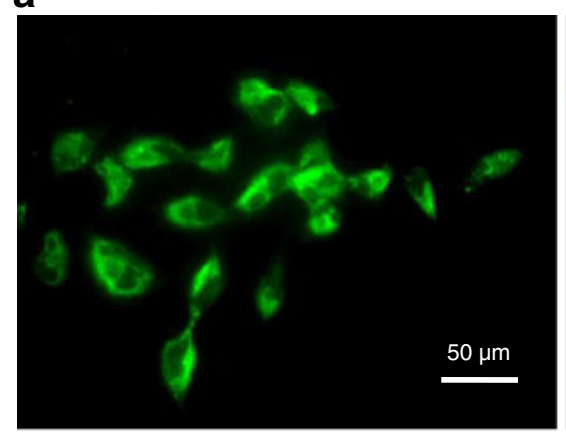

C

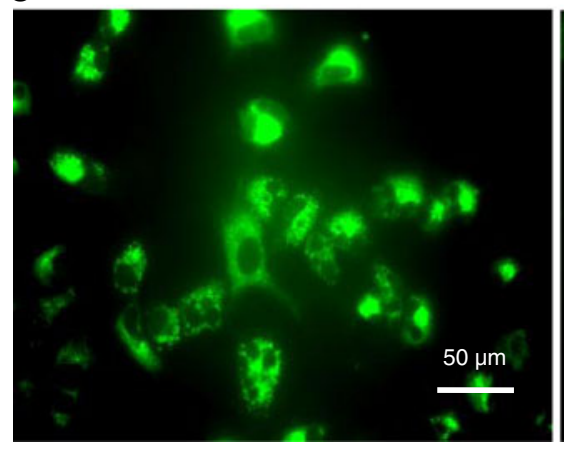

b

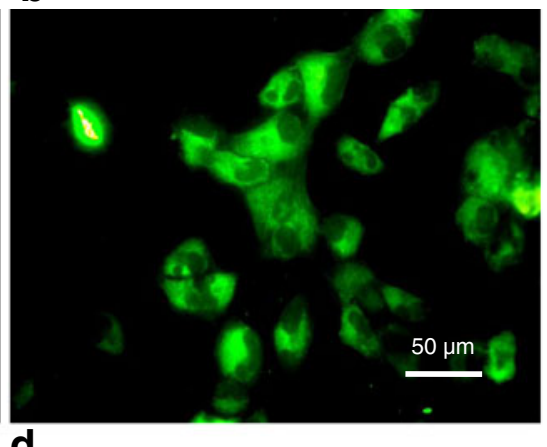

d

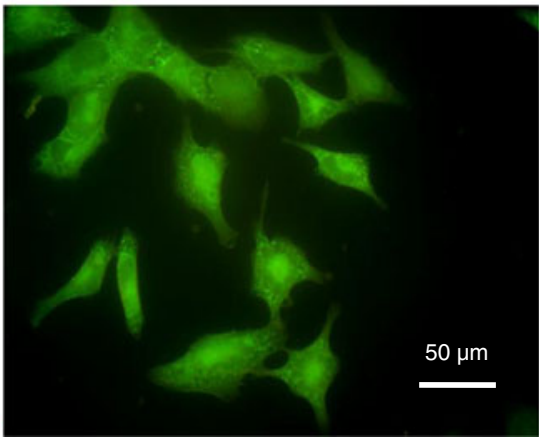

cells were distributed quite uniformly throughout the cytoplasm. After the temozolomide treatment, alone or with quercetin, Hsp27 was localised in association with cytoplasmic round-shaped similar sized granules near the nuclei as well as with the lamellar structures. In the cells with croissant-like nuclei, the protein was located in the curved part of the organelle. In the case of Hsp72, two ways of protein distribution were distinguished. In some cells, the molecular chaperone was arranged in small groups localised around the nuclei and throughout the whole cytoplasm. In the rest of the cells, the localisation of Hsp72 was similar to the control one. Quercetin had no effect on Hsp72 localisation (data not shown).

\section{Discussion}

Most strategies for high grade gliomas are aimed to maximise PCD type I apoptosis or type II autophagy. These are predominantly distinctive processes, but many studies suggest a cross-talk between the two. Apoptosis may occur via death receptor-dependent (extrinsic, cytoplasmic) and receptorindependent (intrinsic, mitochondrial) pathways. The mitochondrial pathway is mediated by $\mathrm{Bcl} 2$ family proteins, which regulate disruption of the mitochondrial membrane and release of cytochrome $\mathrm{c}$ to the cytosol and lead to the activation of caspases and cell death in consequence. The extrinsic pathway is triggered through the activation of death receptors and involves caspase 8 maturation. Both pathways converge to a final common pathway correlated with activation of caspase 3 [4]. Unlike apoptosis, which is a caspasedependent process, autophagy is a caspase-independent programmed cell death, characterised by accumulation of autophagic vacuoles (AVOs) in the cytoplasm accompanied by degradation of Golgi apparatus and ER, which precedes the destruction of the nucleus. Autophagy is suppressed during the early stages of tumour progression. Therapeutically, increased autophagy could represent an alternative way to destroy cancer cells. It would be beneficial in the light of recent investigations, as gliomas naturally resist apoptosis [5, 25]. On the other hand, many studies provide data suggesting that autophagy represents a protective mechanism helping cancer cells to get rid of toxic particles and increase the survival in consequence. In the light of these observations, redirecting cancer cells to enter the apoptotic pathway would be beneficial $[26,27]$. Our earlier experiments conducted on the MOGGCCM anaplastic astrocytoma cell line revealed that temozolomide induced autophagy more efficiently than apoptosis $[15,16]$. We demonstrated for the first time that combinations of TMZ with quercetin were much more effective in programmed cell death induction in glioma cells. At a low $(5 \mu \mathrm{M})$ drug concentration, quercetin potentiated the proautophagic effect of temozolomide, while after treatment with a higher drug concentration $(30 \mu \mathrm{M})$, autophagy switched to apoptosis. Therefore, we decided to evaluate the effect of temozolomide applied alone or in combination with quercetin on induction of apoptosis and autophagy in the human glioblastoma multiforme $\mathrm{T} 98 \mathrm{G}$ cell line. Our results indicated that temozolomide and quercetin were very effective apoptosis inducers in the studied cells. The combination of both drugs 
was even more effective. At the molecular level, it was correlated with the decreased mitochondrial membrane potential, release of cytochrome $\mathrm{c}$ from the mitochondrium to the cytoplasm and activation of caspase 3 and caspase 9. No activation of caspase 8 was observed. All these observations suggest that both drugs initiate apoptosis via the internal (mitochondrial) pathway. In contrast to the MOGGCCM cell line, neither temozolomide alone nor its in combination with quercetin had an effect on autophagy induction in the T98G cells. This was confirmed by the unchanged level of autophagy marker beclin 1 in comparison to the control. This is a critical component involved in autophagosome formation at the early stage of autophagy, which at the basal level of expression is not able to initiate the process of death [28]. It has also been shown that activation of caspase 3 inhibits autophagosome formation and may block beclin 1 activity or even cleave the protein [29]. Furthermore, the expression of beclin 1 is reduced during gliomagenesis, being progressively lower through grade I to grade IV gliomas [30].

Staining the cells with Hoechst 33342 for visualisation of signs of apoptosis additionally revealed that temozolomide alone and together with quercetin changed the morphology of glioblastoma nuclei from circular, observed in the control, to more curved, or 'croissant like'. It is known that tumour cell nuclei belong to the most important histological structures in the assessment of tumour biology including typing and grading of gliomas. Histomorphometry of tumour cell nuclei could also be a promising approach for estimation of the prognosis for individual patients. It was shown that the nuclei had a tendency toward a more circular shape in short survivors [22, 23].

The microscopic observation also revealed small round granules in the cytoplasm after temozolomide treatment, both alone and in combination with quercetin. They were not stained with acridine orange - a fluorochrome typical in the detection of autophagosomes. Staining of cells with $\mathrm{DiOC}_{6}(3)$, a dye that recognises ER within cells, revealed the presence of rounded structures in the reticulum. They were visibly surrounded by Hsp27. This was accompanied by increased caspase 12 expression. All those observations may suggest that this might be correlated with the ER stress response. As tumours increase in size, cells are exposed to several environmental stressors like hypoxia, limited nutrients and acidosis. This leads to accumulation of misfolded and aggregated proteins in the cytoplasm. Such perturbations in protein folding are also observed after exposure to chemotherapy. When molecular chaperones cannot keep up with denaturated and misfolded proteins, they aggregate in larger, uniform in size, membrane free aggresomes present throughout the cytoplasm, leading to ER stress. In response to this, the unfolded protein response is activated. It initially tries to restore normal ER homeostasis by activation of the proteasome complex or autophagy, when aggresomes fuse with lysosome and create autophagolysosomes. In this context, autophagy is a pro-survival process [31]. If damage is too severe, the death pathways are activated especially through the mitochondrial apoptotic pathway [32]. The precise molecular mechanisms of ER stress-induced apoptosis, however, have not been fully elucidated. It is known that ER stress leads to caspase 12 activation [33]. Processed caspase 12 reportedly activates caspase 9 , which is followed by activation of caspase 3 [34]. On the other hand, some reports demonstrate that in fact the mitochondrial pathway of apoptosis plays a significant role in ER stress-induced death, while activation of caspase 12 plays a minor role in the process [35]. In our experiments, the presence of granules and a cleaved form of caspase 12 followed by apoptosis induction was shown in the cells treated with temozolomide alone or with quercetin. In the cells incubated with quercetin, we noticed neither granules nor cleavage of caspase 12 , which may suggest that the ER stress-activated apoptosis in glioma cells after temozolomide treatment is correlated with activation of caspase 12. The lack of ER stress signs in cells incubated with quercetin may be explained by the fact that the antioxidant protects cells from ER stress-induced cell death [36].

Hsps are molecular chaperones whose enhanced expression inhibits ER stress, induces apoptosis and leads to cell survival [32]. The mechanism of Hsp27 and Hsp72 apoptosis blockade is complex. They prevent cytochrome c release, mitochondrial membrane permeabilisation, recruitment of procaspase 9 to the apoptosome and its subsequent maturation to the active form. Hsp 72 migrates to the nucleus to protect nuclear proteins from caspase 3 cleavage. Hsp27 may bind to F-actin, thereby preventing cytoskeleton disruption. It has also been observed that Hsps may be sometimes fused to aggresomes, but they are not directly engaged in misfolded protein degradation. They only recognise them. Depletion of Hsp27 and Hsp72 severely decreases degradation of misfolded proteins and decreases cell survival [31, 32, 37, 38]. All those protective effects of Hsps are beneficial in normal cells. In the case of cancer cells where the level of Hsps is abnormally high, these molecular chaperones act as negative prognostic markers. It was also observed that in many cases, the expression of the proteins rose with the grade of the tumour. It has even been suggested that heat shock proteins may participate in oncogenesis. Therefore, inhibition of Hsps expression has become a novel strategy for cancer therapy [39-41]. In our experiments, both temozolomide and quercetin partially inhibited Hsps expression in T98G cells. 
In summary, our results indicate that temozolomide and quercetin are very potent apoptotic but not autophagic inducers in glioma multiforme T98G cells. It seems that the apoptotic process is preceded by ER stress followed by activation of the mitochondrial death pathway. Our observations may form a basis for further studies on using this drug combination in clinical application.

\section{Conflicts of interest None}

Open Access This article is distributed under the terms of the Creative Commons Attribution License which permits any use, distribution, and reproduction in any medium, provided the original author(s) and the source are credited.

\section{References}

1. Kleihues $\mathrm{P}$ et al. The WHO classification of tumors of the nervous system. J Neuropathol Exp Neurol. 2002;61:215-25.

2. Omuro AM, Faivre S, Raymond E. Lessons learned in the development of targeted therapy for malignant gliomas. Mol Cancer Ther. 2007;6:1909-19.

3. Ohgaki H, Kleihues P. Population-based studies on incidence, survival rates and genetic alterations in astrocytic and oligodendroglial gliomas. J Neuropathol Exp Neurol. 2005;64:47989

4. Ghobrial IM, Witzig TE, Adjei A. Targeting apoptosis pathways in cancer therapy. CA Cancer J Clin. 2005;5:178-94.

5. Lefranc F, Facchini V, Kiss R. Proautophagic drugs: a novel means to combat apoptosis-resistant cancers, with a special emphasis on gliomas. Oncologist. 2007;12:1395-403.

6. Uzzman M, Keller G, Germano IM. Enhanced proapoptotic effects of tumor necrosis factor-related apoptosis-inducing ligand on temozolomide-resistant glioma cells. J Neurosurg. 2007;106:646-51.

7. Kanzawa T, Germano IM, Komata T, Kondo Y, Kondo S. Role of autophagy in temozolomide-induced cytotoxicity for malignant glioma cells. Cell Death Differ. 2004;11:448-57.

8. Tentori L, Graziani G. Recent approaches to improve the antitumor efficacy of temozolomide. Curr Med Chem. 2009; 16:254-7.

9. Roos WP, Batista LF, Naumann SC, Wick W, Weller M, Menck $\mathrm{CF}$, et al. Apoptosis in malignant gliomas cells triggered by temozolomide-induced DNA lesion O6-methyguanine. Oncogene. 2007;26:186-97.

10. Ramos S. Effects of dietary flavonoids on apoptotic pathways related to cancer chemoprevention. J Nutr Biochem. 2007;18: 427-42.

11. Russo GL. Ins and outs of dietary phytochemicals in cancer chemoprevention. Biochem Pharmacol. 2007;22:317-20.

12. Braganhol E, Zamin LL, Canedo AD, Horn F, Tamajusuku AS, Wink MR, et al. Antiproliferative effect of quercetin in the human U138MG glioma cell line. Anticancer Drugs. 2006; 17:663-71.

13. Schültke E, Kamencic H, Zhao M, Tian GF, Baker AJ, Griebel RW, et al. Neuroprotection following fluid percussion brain trauma: a pilot study using quercetin. J Neurotrauma. 2005;22:1475-84.

14. Hosokawa N, Hirayoshi K, Kudo H, Takechi H, Aoike A, Kawai $\mathrm{K}$, et al. Inhibition of the activation of heat shock factor in vivo and in vitro by flavonoids. Mol Cell Biol. 1992;12:3490-8.
15. Jakubowicz-Gil J, Langner E, Rzeski W. Kinetic studies of the effects of Temodal and quercetin on astrocytoma cells. Pharmacol Rep. 2011;63:403-16.

16. Jakubowicz-Gil J, Langner E, Wertel I, Piersiak T, Rzeski W. Temozolomide, quercetin and cell death in the MOGGCCM astrocytoma cell line. Chem Biol Inter. 2010;188:190-203.

17. Jankowska A, Skonieczna D, Rommerts FFC, Warchoł JB. Investigations on apoptosis in Leydig cells cultured in vitro. Folia Histochem Cytobiol. 1997;33:99-110.

18. Takeuchi H, Kondo Y, Fujiwara K, Kanzawa T, Aoki H, Mills GB, et al. Synergistic augmentation of rapamycin-induced autophagy in malignant glioma cells by phosphatidylinositol 3-kinase/protein kinase $B$ inhibitors. Cancer Res. 2005;65:3336-46.

19. Kim EJ, Choi CH, Park JY, Kang SK, Kim YK. Underlying mechanism of quercetin-induced cell death in human glioma cells. Neurochem Res. 2008;33:971-9.

20. Bradford MM. A rapid and sensitive method for quantitation of microgram quantities of protein utilizing the principle of proteindye binding. Anal Biochem. 1976;72:248-54.

21. Laemmli UK. Cleavage of structural proteins during the assembly of the head of bacteriophage T4. Nature. 1970;227:680-5.

22. Nafe R, Franz K, Schlote W, Schneider B. Morphology of tumor cell nuclei is significantly related with survival time of patients with glioblastomas. Clin Cancer Res. 2005;11:21418

23. Nafe R, Franz K, Schlote W, Schneider B. The morphology of perinecrotic tumor cell nuclei in glioblastomas shows a significant relationship with survival time. Oncol Rep. 2006;16:55562.

24. Terasaki M, Reese TS. Characterization of endoplasmic reticulum by co-localization of BiP and dicarbocyanine dyes. J Cell Sci. 1992;101:315-22.

25. Lefranc F, Kiss R. Autophagy, the Trojan horse to combat glioblastomas. Neurosurg Focus. 2006;20:E7.

26. Lin CJ, Lee CC, Shih YL, Lin CH, Wang SH, Chen TM, et al. Inhibition of mitochondria- and endoplasmic reticulum stressmediated autophagy augments temozolomide-induced apoptosis in glioma cells. PLoS One. 2012;7:e38706.

27. Garcia-Arencibia M, Hochfeld WE, Toh PPC, Rubinsztein DC. Autophagy, a guardian against neurodegeneration. Semin Cell Dev Biol. 2010;21:691-8.

28. Wang J. Beclin 1 bridges autophagy, apoptosis and differentiation. Autophagy. 2008;4:947-8.

29. Luo S, Rubinsztein DC. Apoptosis blocks Beclin 1-dependent autophagosome synthesis - an effect reduced by Bcl-xL. Cell Death Differ. 2010;17:268-77.

30. Pirtoli L, Cevenini G, Tini P, Vannini M, Oliveri G, Marsisli S, et al. The prognostic role of Beclin 1 protein expression in high-grade gliomas. Autophagy. 2009;5:930-6.

31. Rodriguez-Gonzales A, Lin T, Ikeda AK, Simms-Waldrip T, Fu C, Sakamoto KM. Role of the aggresome pathway in cancer: targeting histone deacetylase 6-dependent protein degradation. Cancer Res. 2006;68:2557-60.

32. Gupta S, Deepti A, Deegan S, Lisbona F, Hetz C, Samali A. Hsp72 protects cells from ER stress-induced apoptosis via enhancement of ire $1 \alpha$-xbp 1 signaling through a physical interaction. PLoS Biol. 2010;8:e1000410.

33. Nakagawa T, Zhu H, Morishima N, Li E, Xu J, Yankner BA, et al. Caspase 12 mediates endoplasmic-reticulum-specific apoptosis and cytotoxicity by amyloid-beta. Nature. 2000;403:98103.

34. Rao RV, Castro-Obregon S, Frankowski H, Schuler M, Stoka V, del Rio G, et al. Coupling endoplasmic reticulum stress to the cell death program. An Apaf-1-independent intrinsic pathway. J Biol Chem. 2002;14:1836-42. 
35. Shiraishi H, Okamoto H, Yoshimura A, Yoshida H. ER stressinduced apoptosis and caspase-12 activation occurs downstream of mitochondrial apoptosis involving Apaf-1. J Cell Sci. 2006;119:3958-66.

36. Mauro C, Crescenzi E, De Mattia R, Pacifico F, Mellone S, Salzano $\mathrm{S}$, et al. Central role of the scaffold protein tumor necrosis factor receptor-associated factor 2 in regulating endoplasmic reticulum stress-induced apoptosis. J Biol Chem. 2006;281:2631-8.

37. Stetler RA, Gan Y, Zhang W, Liou AK, Gao Y, Cao G, et al. Heat shock proteins: cellular and molecular mechanisms in the CNS. Prog Neurobiol. 2010;92:184-211.
38. Turturici G, Sconzo G, Geraci F. Hsp70 and its molecular role in nervous system diseases. Biochem Res Int. 2011;2011:618127.

39. Garrido C, Brunet M, Didelot C, Zermati Y, Schmitt E, Kroemer G. Heat shock proteins 27 and Hsp 70: anti-apoptotic proteins with tumorigenic properties. Cell Cycle. 2006;5:2592-601.

40. Sreedhar AS, Csermley P. Heat shock proteins in the regulation of apoptosis: new strategies in tumor therapy. A comprehensive review. Pharm Ther. 2007;101:2227-57.

41. Graner MW, Cumming RI, Binger DD. The heat shock response and chaperone/heat shock proteins in brain tumors: surface expression, release and possible immune consequences. J Neurosci. 2007;27:11214-27. 